Voix et Images

volxetimages

\title{
Vers une mythologie de la renaissance : le Saint-Elias
}

\section{Neil B. Bishop}

Volume 8, numéro 3, printemps 1983

Jacques Ferron

URI : https://id.erudit.org/iderudit/200401ar

DOI : https://doi.org/10.7202/200401ar

Aller au sommaire du numéro

\section{Éditeur(s)}

Université du Québec à Montréal

ISSN

0318-9201 (imprimé)

1705-933X (numérique)

Découvrir la revue

Citer cet article

Bishop, N. B. (1983). Vers une mythologie de la renaissance : le Saint-Elias. Voix et Images, 8(3), 456-464. https://doi.org/10.7202/200401ar d'utilisation que vous pouvez consulter en ligne.

https://apropos.erudit.org/fr/usagers/politique-dutilisation/ 


\section{Vers une mythologie de la renaissance: lo Saint-Elias}

par Neil B. Bishop. Université de Victoria.

Pour A. Renaud, "Ferron, voulant guérir notre belle province des plaies de sa traditionnelle mythologie, tente d'en créer une nouvelle, mieux conforme, selon iui, au fond réel et historique de notre collectivite"'. En effet, le Saint-Elias utilise lè terme "mythologies" en évoquant l'évolution de celles-ci"; en outre, le personnage de Mithridate III, jumeau fictif (un médecin qui fait des livres pendant ses loisirs) du docteur Ferron, valorise cette fonction de transformateur de mythologies: "J'écris et je refais la réalité de mon pays à mon gré" (p. 185). II s'agira pour nous de préciser en quoi le Saint-Elias contribue à renouveler la mythologie québécoise, mythologie à la fois littéraire et sociale (car tout mythe littéraire est un fait sociai): dans ce but nous tenterons de dégager les mythes que véhicule ce roman, et de dégager le caractère et la portée mythologiques - et mythogènes - de plusieurs de ses composantes, dont ce navire-symbole qui lui donne son titre, ainsi que les personnages principaux constitutifs d'un univers humain romanesque fort novateur et signifiant.

Le grand mythe fondamental que le Saint-Elias met en roman est celui du pays nation (ou pays-peuple). L'important discours que prononce le chanoine Tourigny lors du lancement du Saint-Elias vise à préciser "qui nous sommes, nous, les gens de Batiscan" (p. 15). Cette définition d'un peuple se fait en termes d'espace - il s'agit des gens de Batiscan - et de temps, car Tourigny dresse d'abord une synthèse de l'his toire des Batiscanais depuis la Conquête ("Après le départ des Français", p. 16), jusqu'à l'époque du lancement du Saint-Elias ("1869", p. 19), pour aborder enfin des perspectives d'avenir. Or, les "gens de Batiscan" sont clairement, dans ce roman, à la fois la synecdoque et le symbole du peuple québécois à venir lou à faire). La conclusion du discours de Tourigny affirme cette définition de paysnation au sens d'un peuple ayant une histoire (nonobstant Durham), habitant un espace sien, si riche d'un projet d'avenir collectif. Cette vision d'un avenir positif, suite aux "empêchements de l'enfance" qui suivirent la Conquête, constitue le premier élément d'une mythologie de la renaissance:

"Vous me demanderez pourquoi nous I" (le Saint-Elias) avons construit. Je vous répondrai que ç'a été pour briser l'écrou de notre pays. (...) que soit brisé l'écrou du Golfe! que cessent les empêchements de l'enfance! Nous avons băti le SaintElias pour aller au-delà de Terre-neuve, dans le grand océan, vers les Bermudes 
et les Antilles, au besoin vers les vieux pays... Qui sommes-nous, gens du Batiscan? Les égaux des Maloins, capables de découvrir l'Europe (...) plus rien ne nous entravera; nous serons libres, nous serons gens de toutes les mers du monde" (p. 19).

Une caractéristique du pays-nation, valorisé par ce roman, sera l'unité de sa population: en témoignent des formules comme "nous, gens du Batiscan". "mes amis, mes frères" (p. 150), les grands rassemblements provoqués par le lancement du navire et les funérailles de Fauteux, l'unité des Batiscanais (toutes classes confondues) lors de leur épreuve de force avec l'autorité extérieure (Mgr Laflèche, p. 144-145), et les origines mixtes de certains personnages importants (Fauteux; Marguerite Cossette et ses descendants). Ce roman valorise ainsi le mythe de l'unité nationale, comme le suggère la remarque de Renaud concernant le rapprochement, par Ferron, $d$ "'institution"et de "groupes sociaux très différenciés chez nous" 3 .

Le mythe foridamental du pays-nation - véritable mythe-projet ${ }^{4}$ - structure toute l'organisation mythologique du roman, les autres mythes venant renforcer le mythe-projet de base. Les autres mythes qu'engendre le Saint-Elias sont dans un rapport d'interdépendance entre eux et avec le mythe du pays-nation. L'élément textuel le plus apte à nous permettre d'aborder et de mieux comprendre le riche tissu mythologique du roman est bien le Saint-Elias lui-même.

Le trois-mâts - symbole, comme l'indiquent les propos de Tourigny (p. 19), d'un esprit de dynamisme et d'ouverture - acquiert au cours du roman le statut d'objet mythique. Le fait de construire le Saint-Elias répondait, selon Tourigny (p. 16-19) et l'armateur même, Philippe Cossette (p. 150), au malheur de l'enfermement qui suivit la Conquête: le navire participe ainsi, dès sa construction, d'une mythologie de la renaissance d'un peuple. Le testament de Tourigny participe de cette même mythologie et témoigne d'une conscience aigué du potentiel symbolique et mythique du SaintElias: "La gloire et la beauté sont patientes: quand elles ont vu le jour, elles savent attendre parce que l'avenir leur appartient" (p. 164). Cette gloire, cette beauté sont celles du Saint-Elias; le testament de Tourigny assure au trois-måts un dernier "voya gement pour la beauté, pour qu'une dernière fois à Batiscan revienne ce bătiment, toutes voiles déployées" (p. 164). "Un voyagement", précise le testament, "de principe" - c'est-à-dire, symbolique. Ce dernier voyage, en ancrant le navire dans la mémoire et l'imaginaire des Batiscanais, lui permettra de mieux accéder au statur d'objet mythique.

Le lancement fournit a Tourigny l'occasion de définir le pays-nation en termes d'espace et de temps. De méme, le Saint-Elias contribue à cette définition du pays, aussi bien dans l'espace que dans le temps. Cette contribution consiste, aux deux plans, en une activité unifiante permettant de rapprocher: a) les Batiscanais entre eux (la construction et les voyages du navire sont présentés par Tourigny (p. 19) comme par Cossette (p. 150) comme ayant été le fait d'un "nous", d'un sujet collectif composé de tous les Batiscanais); b) les Batiscanais avec d'autres Québécois, d'une part, et aussi avec d'autres francophones; c) ces Batiscanais romanesques de 1869 et les Québé cois du hors-texte de l'époque de la publication du roman (1972). 
Absents lors du lancement du Saint-Elias (p. 11), les espaces proches et leurs habitants seront progressivement reliés à Batiscan et, d'une rive a l'autre, les uns aux autres, puisque, le long des deux rives du fleuve, les yeux, comme les esprits, remarquent le trois-mâts qui rattache ainsi à Batiscan tout l'Est de ce Québec romanesque. Or, au dix-neuvième siècle, plus encore que maintenant, l'Est du Québec était plus francophone que l'Ouest (Montréal et ses environs) ${ }^{5}$. Se définit ainsi l'espace mythique romanesque qui, tout en étant celui de l'Est québécois, est aussi - puisqu'inscrit dans un roman - fictif, celui donc de ce "pays qui avait été et serait devenu mythique" (p. 181) Itel cet "Erewhon" de S. Butler dont le nom en anagramme et la localisa tion vague confirme le caractère mythique et utopiquel. L'espace batiscanais/québécois apparaît néanmoins comme étant aussi celui qui - en retrouvant l'esprit du SaintElias, on le verra - pourrait se remettre à exister et à s'épanouir.

Le Saint-Elias contribue aussi à l'edification du mythe d'un pays-nation francophone, ayant avec d'autres espaces et peuples francophones des rapports privilégiés. Le roman signale le caractère francophone des pays que le navire relie à Batiscan: le Sénégal ("dont les habitants d'eux-mêmes se nomment Canadiens", p. 116), les Antilles françaises ("Cap Haïtien", p. 61), La Rochelle (p. 61) ("Dans ces vieux pays-là, vous le croirez peut-être pas, on parle quasiment comme ici, en français", dit un matelot, p. 60). Le mythe d'une renaissance québécoise, dans ce roman, a partie liée avec le mythe des retrouvailles des Québécois avec leurs racines francophones.

La contribution du Saint-Elias au mythe du pays-nation opere aussi dans le temps. Le trois-máts traverse le temps, car il sera proposé comme instrument de salut au dernier rejeton de la dynastie des Mithridate-Cossette (p. 186) et rapprochera ainsi sa génération des Batiscanais de l'époque du lancement (1869), voire des fondateurs de la Nouvelle-France, ces "Maloins" (p. 19), navigateurs animés par cet esprit de dynamisme que symbolise le Saint-Elias. Le navire aide ainsi les Batiscanais/Québécois à franchir la faille historique de la Conquête, après laquelle, selon Tourigny, les Canadiens français se sentaient incapables de navigations océaniques (p. 16-17): le Saint-Elias, en durant, est à même d'aider une génération nouvelle à reprendre en main son destin. Par là, ce navire fictif renforce l'inscription romanesque du mythe-projet d'une renaissance collective, c'est la le sens des propos de Marguerite Cossette qui terminent le roman, tout en louvrant sur un avenir possible: Marguerite recommande que le dernier rejeton de la dynastie des Mithridate-Cossette prenne "possession du monde " en retournant à Batiscan pour y trouver le Saint-Elias - seule "façon d'échapper à son désastre" (ce désastre: la ruine de la dynastie Mithridate-Cossette, métaphore de la Conquête de 1763).

Lors de la dernière évocation du Saint-Elias, dont le nom termine le roman, se fixant ainsi dans l'imaginaire de Mithridate IV comme des lecteurs du roman, ce navire existe-t-il encore "réellement" (dans le réel fictif) ou seulement dans les souvenirs de la vieille Marguerite? Elle-même a affirmé que le navire avait été brûlé (p. 178). Qu'importe: le vaisseau survit, puisque l'on en parle; l'objet absent, on le nomme néanmoins, on l'appelle, on en signale la fonction salvatrice possible et espérée. C'était bien le but du testament de Tourigny en assurant au navire un dernier "voyagement" symbolique et mythogène. Mué en objet du discours utopique, avant perduré au-delà même 
de son existence concrète, le trois-mâts a conquis le statut du mythe, un de ces objets de langage qui font vivre les hommes.

Certaines cargaisons du Saint-Elias contribuent à la création, dans ce roman, d'une mythologie de la renaissance. L'idole sénégalaise que rapporte le navire (p. 116) participe à la remise en cause des valeurs catholiques traditionnelles et relaie la portée psychologique et mythique du navire: enrichissement du vécu symbolique des Batiscanais, fraternité entre peuples francophones. En plus de symboliser la pluralité des croyances et des valeurs socio-culturelles, l'idole introduit symboliquement, dans l'aire batiscanaise, la sexualité et le désir comme en témoignent les nombreuses allusions à sa nudité et à ses mamelons.

L'idole subira une agression (amputation des seins, p. 163) traduisant sans doute le caractère frustré et maladif de la sexualité dans le Québec de Mgr Laflèche. Important est le sort que Tourigny réservera alors à l'idole, car elle sera promue sans ambiguîté au rang d'objet mythique dans une mythologie que le texte romanesque identifiera explicitement comme en étant une de la renaissance: "Qu'elle soit brûlée à l'embouchure de la rivière Batiscan et du fleuve Saint-Laurent, du côté de l'église. De ses cendres renaîtront la beauté et la force dans notre paroisse (p. 163, nous soulignons)." Même le rapprochement spatial, entre l'église et le lieu où doit brûlèr l'idole, importe, comme si la mythologie nouvelle devait surgir grâce à la fécondation de la mythologie ancienne (représentée ici par "l'église") par un apport nouveau, de provenance étrangère: nous y reviendrons.

A la fois universelle et québécoise, la mythologie ferronienne est nouvelle par comparaison à une certaine mythologie québécoise traditionnelle. Le Saint-Elias innove par rapport à nombre de romans québécois antérieurs par la gamme de personnages qu'il met en texte. Ce roman est moins "populiste" que maints romans québécois: la part faite au peuple y est bien plus restreinte que dans d'autres romans québécois a forte coincidence texte-hors-texte ${ }^{6}$. Tout se passe comme si, pour Ferion, l'on avait suffisamment mis en roman des personnages populaires écrasés, un peuple canadienfrançais bloqué, et qu'il était temps de présenter au public lecteur québécois des personnages d'un type nouveau, représentạnt une élite motrice potentiellement salvatrice, et d'élargir ainsi la mythologie québécoise.

Un tel point de vue expliquerait l'importance du personnage de l'homme d'affaires Philippe Cossette/Mithridate I. Les hommes richies ne manquent pas en littéra ture québécoise, mais il ne s'agit ici ni, par exemple, du "seigneur" propriétaire terrien des Anciens Canadiens, ni du riche paysan avare d'Un homme et son péché, ni de I'homme d'affaires allié-pion des Anglophones d'Hier les énfants dansaient. Cossette a réussi de façon indépendante, libre de l'aide comme de la domination des Anglophones, voire en refusant de parler anglais (p. 185). L'importance et la dimension innovatrice du personnage ont partie liée avec son statut socio-économique: bourgeois, Cossette peut offrir aux Batiscanais ce navire-symbole qui permet l'inscription roma nesque du mythe d'un peuple animé d'un esprit d'initiative et de dynamisme, et celui d'un peuple uni jouissant de rapports égalitaires avec d'autres peuples, rapports constitutifs - déjà - d'une proto-souveraineté collective. Ce bourgeois innove aussi en traitant ses employés (à qui il rend hommage, p. 150) de façon égalitaire, à en juger 
par le fait qu'ils le tutoient (p. 149): cet égalitarisme consolide le mythe de l'unité sociale et nationale.

L'importance de Philippe Cossette découle surtout du fait que le roman lui attribue l'un des grands discours énonciateurs du mythe-projet d'un pays-nation épanoui:

"(...) le bateau (...) porte le nom de mon pays. (...) lci, nous sommes libres et entreprenants. (...) On nous avait enfermés dans notre pays. (...) Par ce trois-mâts (...) nous avons rompu les barreaux de notre pays, nous avons repris contact avec le monde. (...) Les hommes d'un village, des Batiscanais, et nous sommes rendus plus loin que les gens des villes (...) qui, dominés par les étrangers, attendent des étrangers ce qu'ils pourraient faire eux-mêmes." (p. 150)

Le syntagme "nous avons repris contact avec le monde" (nous soulignons) confirme que ce discours participe' d'une mythologie de la renaissance. Ce discours confirme fortement la signification du refus par Cossette de parler anglais. $\dot{A}$ travers le personnage de Philippe Cossette, c'est toute une classe sociale, une bourgeoisie d'affaires patriote, québécoise, économiquement motrice, socialement dynamisante, qui trouve texte et se valorise par sa participation à l'élaboration du mythẹ-projet fondamental. La valorisation de cette classe sociale dans le cadre du mythe-projet de base est confirmée par l'attribution à Philippe et à ses descendants du surnom "Mithridate", le Mithridate historique ayant, selon Tourigny, "passé sa vie à lutter contre les Romains, qui étaient les Anglais du temps" (p. 55). L'attribution de ce surnom mélioratif à Philippe confirme la valeur mythique du personnage.

Les médecins ne sont pas rares en littérature québécoise, mais le docteur Fauteux, athée humaniste, innove par sa décision de se suicider et, surtout, de faire par un écrit - de son suicide un geste symbolique, potentiellement générateur d'un nouveau type de rapports, plus égalitaires, entre notables et peuple. En décidant d'être enterré parmi les "fous" et les "pauvres misérables" du champ du Potier (p. 113, 126), le notable Fauteux consolide le mythe de l'unité nationale en favorisant le rassemblement d'un pays-nation batiscanais/québécois, toutes classes réunies. Sur un plan plus général, Fauteux, en souscrivant à des valeurs autres que celles de la société québé coise traditionnelle, contribue à la mise en roman d'une nouvelle mythologie québécoise.

Le personnage de Fauteux permet aussi à un autre mythe important de prendre texte dans /e Saint-Elias: le mythe d'une capacité québécoise d'assimiler les immigrants. Faustus, l'ancêtre allemand de Fauteux, a suivi un processus typique d'assimilation: difficultés d'adaptation initiales, rencontre avec une autorité locale ('le grand vicaire Saint-Onge", p. 26) qui, sans rudesse mais avec fermeté, lui a fait prendre le chemin d'une assimilation définitive - apprentissage du français, mariage avec une fille du pays, vie de famille, fondation d'une lignée dont fauteux est l'aboutissement (p. 26-28). Ce même mythe de la capacité québécoise d'assimiler les immigrants se manifeste à travers l'Irlandais Jos Magloire, assimilé au point de proférer des jurons du pays (p. 121). Comme l'écrit G. Marcotte de l'oeuvre de Ferron, "Quant aux étrangers, Irlandais, Ecossais, Allemands, on les enquébéquoise en deux temps trois mouvements, de gré ou de force"7. La forte présence de ce mythe dans le Saint-Elias est d'autant plus significative que, vers l'époque de la publication du roman, battait son plein le débat sur la manière dont la société québécoise devait aborder le problème 
posé par les immigrants qui tendaient à s'angliciser. La fermeté du vicaire Saint-Onge prend, dans ce contexte, valeur de modèle.

Dans le cadre général du mythe-projet de l'édification d'un paysnation, ce mythe de l'assimilation des immigrants à la collectivité québécoise revêt lui-même - comme les autres mythes du roman - un caractère de mythe-projet. Ce mythe ne réduit pas pour autant l'immigrant à la fonction passive de l'assimilé sans résonance dans la collectivité d'accueil: la preuve en est. la fonction d'iconoclaste, de transformateur des valeurs, qui est celle de Fauteux. L'immigrant véhicule la possibilité de renouveler les valeurs culturelles et la mythologie québécoise, de sorte que G. Marcotte, qui souligne la tendance à l'enquébécoisement de l'immigrant chez Ferron, a raison d'écrire que la création de mythes dans cette oeuvre passe par l'étranger enquébécoisé. "Comme si le village québécois ne pouvait renaître que par la rencontre de l'autochtone et de l'étranger"9. Rappelons à ce sujet nos propos sur la signification mythogène de l'idole sénégalaise et de son lieu de combustion.

Le chanoine Tourigny, personnage principal du roman, n'est guère moins iconoclaste que son ami Fauteux, et sa position au centre de la collectivité batiscanaise assure à ses gestes et paroles une résonance signifiante plus grande. Non content de bafouer l'autorité d'un haut clergé trop ami des ennemis anglo-saxons de la collectivité batiscanaise/québécoise (p. 148), Tourigny, en improvisant pour Fauteux des funé railles qui s'écartent nettement du rite funéraire catholique, écrit en paroles et en actions un rite et un code nouveaux (chapitre XVIII). Ces funérailles se fondent moins sur des valeurs religieuses que sur d'autres valeurs mythiques: la fraternite et un humanisme laic. Le Dieu traditionnel des Québécois est exclu de ce nouveau rituel et de la mythologie qu'il implique. En mettant en cause les valeurs du catholicisme et en promouvant un nouvel ensemble de valeurs humanistes, Tourigny tend a mener son immense auditoire vers une tolérance et un pluralisme des croyances caractéristiques d'un Etat laîc et libéral éloigné de ce que le Québec traditionnel avait de théocratique.

Peut-être bien est-ce Marguerite Cossette le personnage le plus important du Saint-Elias du point de vue de son écart par rapport aux personnages traditionnels du roman québécois: grande, donc, est sa capacité de contribuer à l'élaboration d'une nouvelle mythologie québécoise. Du personnage féminin traditionnel il reste l'amour maternel - mais ce trait est renouvelé par une dimension incestueuse assez explicite: à son fils, "malgré quelques passades, elle fut fidèle"; quant à l'âge de le marier, "cet âge, elle l'aurait remis durant un siecle" (p. 174). Marguerite est plus encore le personnage de la libertine, d'abord pour s'assurer un enfant, mais aussi pour son plaisir. Prendre un abbé comme amant constitue déjà un travail de sape à l'égard des valeurs traditionnelles; Marguerite met fin à sa liaison avec Lupien avec comme seul mobile sa propre convenance - et ne se prive pas de prendre d'autres amants (p. 158). Marguerite incarne ainsi, dans cette mythologie romanesque, la femme désirante et la liberté sexuelle féminine. Point nymphomane pour autant, elle agit par choix et par volonté, comme l'indiquent sa rupture avec l'abbé Lupien, son coît unique avec le curé Rondeau (acte qui poursuit la subversion des valeurs traditionnelles, p. 172-173), et sa fidélité envers son fils. Triomphante en amour, elle est aussi une femme d'affaires habile, type de personnage particulièrement novateur. Par le rôle que jouent dans sa vie l'intelligence, la volonté, l'esprit de conquête et l'érotisme, Marguerite intègre ces valeurs 
dynamisantes au mythe-projet d'un pays renouvelé: à l'édification du pays-nation elle intègre la femme. En énonçant le message-conclusion du Saint-Elias (c'est elle qui a, littéralement, le dernier mot) Marguerite - et le mythe d'un nouveau type féminin - se campe au centre du processus de renouvellement mythologique, puisqu'elle permet la jonction du passé et de l'avenir.

Le mythe-projet qu'énonce ce roman repose sur une structure roman : hors-texte $=$ microcosme : macrocosme.

Chacun des deux éléments microcosme/macrocosme obéit à une évolution inverse lqui renforce la pertinence des préfixes "micro" et "macro"l. Le microcosme que forment Batiscan et sa population se trouve, à la fin du Saint-Elias, condensé dans le seul personnage du "pauvre petit homme", dernier de la dynastie batiscanaise des MithridateCossette, Mithridate IV, à qui s'adresse le message-conclusion de Marguerite. Ce même Mithridate IV, toutefois, selon une démarche en expansion et gråce aux multiples origines (dont l'amérindienne) ${ }^{10}$ de Marguerite, ainsi qu'à la provenance extrabatiscanaise de. Lupien, sert d'embrayeur vers le macrocosme, qui en vient à se composer de tout le peuple québécois, appelé dans les dernières pages du roman à lutter contre sa dépos session. Oméga du microcosme batiscanais, le petit Mithridate IV est aussi l'alpha d'un macrocosme québécois à venir lou à fairel. Tout comme il existerait pour Mithridate IV un espoir et une chance de salut, il en existerait pour le peuple québécois - pourvu qu'a l'instar de Mithridate IV ce peuple tout entier retrouve l'esprit du Saint-Elias, adhère a cette nouvelle mythologie au centre de laquelle trone le beau mythe du trois-mats, mythe de l'initiative" et de la renaissance.

Nombreux sont ainsi, dans le Saint-Elias, les images et les personnages contribuant au renouvellement de la mythologie littéraire québécoise. Cette mythologie roma nesque qui propose, par Batiscan interposé, un Québec dynamique et confiant, dialoguant avec les autres pays peuples sur un pied d'egalité, s'oppose à trois grands mythes traditionnels du Québec. P. L'Hérault voit juste en affirmant que 'I'oeuvre de Ferron rend aux Québécois leur véritable histoire, en la libérant de ses tendances à la glorification et au défaitisme" ${ }^{\prime 12}$; tentons de compléter et de préciser sa formule.

La mythologie du Saint-Elias s'oppose, d'une part au messianisme fréquent dans I'historiographie et la littérature québécoisés, messianisme qui impliquait que le peuple québécois était en quelque 'sorte supérieur aux autres ${ }^{13}$. La mythologie qu'écrit le Saint-Elias propose, au contraire, un rapport d'égalité entre le peuple québécois et les autres. Par là, ce roman s'oppose également à un deuxième grand mythe québécois, celui de la survivance, mythe statique d'un peuple replié sur lui-même et sur la défensive et impliquant que ce peuple était inférieur aux autres. Le Saint-Elias s'oppose au mythe de la survivance, et par sa valorisation de rapports égalitaires entre les pays-peuples, et par son affirmation de la capacité québécoise d'assimiler les immigrants, et par son insistance sur la possibilité d'un avenir dynamique comportant non seulement une survivance mais l'épanouissement ${ }^{14}$.

Ce roman bouleverse, troisièmement, le mythe canadien-français de la supériorité du passé sur l'avenir, mythe inhérent à l'idéologie du "Notre Maître le passé", qui impliquait l'assujettissement du présent et de l'avenir au passé et à ses modèles de société. Tout autre est la conception du passé dans le Saint-Elias: certes, pour pou- 
voir savoir qui est et se définir en tant que pays-nation un peuple doit connaitre son histoire, en témoigne l'insistance que met Tourigny (p. 16-19) à raconter ce que A. Th. Reilly nomme, justement, "I'histoire (de) la tribu" 15. Mais le discours de Tourigny aboutit à des perspectives d'avenir en vue d'une collectivité renouvelée grace au SaintElias: aucune inféodation, dans ce discours, de l'avenir au passé; l'avenir sera non pas la fidélité figée au passé, mais autre chose, du nouveau. Le narrateur lui-même affirme cette prééminence de l'avenir sur le passé:

On ne saurait finir dans le passé car le temps n'a qu'un mouvement; il vient du passé, passe par le présent et va vers l'avenir. On ne remonte pas à l'ancien, on en repart (...) (p. 181).

L'utilisation dynamique du passé ldont la valorisation des Maloins dans les discours que prononce Tourigny lors du lancement du trois-mâts constitue 'un exemplel remplace le mythe de la fidelité à une image figée du passé par le mythe de l'esprit d'initiative, celui du Saint-Elias. La valorisation du présent et de l'avenir dans une formule de Lupien ("le Fils (...) qui engendre le Père et commence le règne de Dieu", p. 183) ainsi que celle de l'avenir dans le testament de Tourigny ("La gioire et la beauté (...) savent attendre parce que l'avenir leur appartient", p. 164) vont dans le même sens.

Ce roman de la mythologie nouvelle tend à rapprocher réel et fictif, texte et hors-texte. Il est pourtant loin d'obéir à une esthétique réaliste; en témoignent le caractère a-réaliste de sa gamme de personnages et le rapprochement qu'il effectue entre "institutions" et "groupes sociaux très différenciés" 16 dans la réalité hors-textuelle: la société québécoise de ce roman est "utopisante", proposée non pas comme reflet de la vraie, mais comme mythe. De tels procédés scripturaux confirment qu'au contraire d'une esthétique réaliste qui chercherait à faire ressembler une fiction à la réalité, l'esthétique romanesque du Saint-Elias vise à faire ressembler la réalité à la fiction, à transformer celle-là au profit de celle-ci et, par là, engendrer une réalité nouvelle ${ }^{17}$ - voilà le but même, à n'en pas douter, de la création d'une nouvelle mythologie québécoise dans ce roman.

Une réflexion sur l'écriture mythologique et mythogène du Saint-Elias se doit de prendre en compte ses références explicites au mythe de Faust. Le mythe germanique est évoqué surtout à propos du docteur Fauteux dont l'ancêtre allemand s'appelait Faustus, nom dont "Fauteux" est une variante enquébécoisée, pour reprendre le terme de G. Marcotte. L'utilisation du mythe de Faust - dont Fauteux, par son suicide même, reconnaît la pertinence (p. 113) malgré ses dénégations antérieures (p. 110-111\} - signifie que les mythes d'ailleurs, et par conséquent tout l'apport européen, sont à intégrer à l'expérience québécoise - à condition de les adapter (Fauteux, par exemple, correspondrait plutót à Méphisto qu'a Faust) à la réalité québécoise sans tenter de déformer celle-ci au profit de schémas venus d'ailleurs. Nul projet de renaissance nationale sans introjection collective et bénéfique du passé, européen et amérindien comme québécois, mythique comme historique.

L'intégration du mythe de Faust à ce roman ecrit par un grand conteur traduit aussi, à notre sens, l'intention esthétique de rapprocher ce roman de ces genres plus antiques et universels que sont les contes et les mythes. "Refaire la réalité de (s)on pays" (p. 185) relève d'une écriture de l'ici mais non d'une littérature régionaliste: La 
juxtaposition de mythes anciens (Faust) et nouveaux (le Saint-Elias, le curé humaniste et nationaliste, le Québécois homme d'affaires, la fraternité francophone, la fernme désirante et femme d'affaires, et nous en passons) réussit la synthèse d'une activité créatrice de type ancien. (création de mythes) et d'une écriture de type moderne (le roman et, plus moderne encore, le roman engagél. En faisant délibérément d'un roman quô. bécois un objet culturel générateur de mythes, Ferron confirme à la littérature québécoise son statut de littérature de portée universelle.

Férron, à l'encontre de nombre de ses contemporains, ne fait pas de l'écriture l'objet principal de son roman, ne raconte pas un livre en train de s'écrire - mais nous fait assister à l'histoire d'un pays en train de se (relfaire par des livres, dont le SaintElias lui-même: "J'écris et je refais la réalité de mon pays à mon gré" dit le médecinécrivain Mithridate III (p. 185). II s'agit là encore d'un mythe, tout aussi intéressant que les autres de ce roman, mythe de l'écrivain à même d'influer sur sa société, de participer au façonnage (le Saint-Elias comme le livre qui lui donne texte et vie étant, dans le contexte de l'édification d'un pays-nation, des "produits utiles" tirés "des arbres abattus" $)^{18}$ du devenir collectif. Voilà un mythe auquel l'on avait quelque peu perdu foi. L'intérèt du Saint-Elias en est rehaussé d'autant. Déjà, de par sa dimension mythogène, ce roman constitue une oeuvre à résonances contemporaines. II confirme, en véhiculant ce dernier mythe, celui (point neuf, certes, mais d'une intense actualité) de l'écrivain comme agent social efficace par son écriture même, sa forte pertinence dans les débats présents sur la "crise de la littérature québécoise" - et sa raison d'être fondamentale, qui est de mettre en texte une mythologie de l'espoir, celui d'une renaissance collective.

1. André Renaud, "La Chaise du maréchal ferrant et le Saint-Elias de Jacques Ferron", Liures et Auteurs québécois 1972, p. 42.

2. Jacques Ferron, le Saint-Elias (roman), Montréal, Editions du Jour, 1972, p. 183. Désormais, les références paginales seront précisées entre parenthèses dans notre texte.

3. A. Renaud, op. cit., p. 44.

4. La dimension "projet" des mythes et de la mythologie ferroniens correspond bien à l'une des définitions du mythe que donna G. Sorel: "Image d'un avenir fictif (...) qui exprime les sentiments d'une collectivité et sert à entrainer l'action" 'icité dans Paul Robert, Dictionnaire alphabétique et analogique de la langue française, Paris, Société du Nouveau Littré, 1959. tome 4, p. 723).

5. Voir Jean-Claude Robert, Du Canada français au Québec libre, Flammarion, coll. "I'Histoire vivante", 1975 p. 102-103, 146. Cf. aussi P. L'Hérault pour qui, chez Ferron, "on ne va jamais vers l'ouest. $Y$ aller, $c$ 'est aller vers la mort (...) les forces de résistance et de libération (...) ouvrent par le fleuve un espace libre d'entraves, l'espace de l'Est" Idans son excellent Jacques Ferron, cartographe de l'imaginaire, Montréal, P.U.M., coll. "Lignes québécoises", 1980, p. 64-65.

6. Cf. Robert Migner, "Jacques Ferron et l'histoire de la formation sociale québécoise", Etudes françaises, vol. XII, no $3-4$ loctobre 1976), p. 348.

7. Gilles Marcotte, "Jacques Ferron, côté village", Ėtudes françaises, vol. XII, no 3-4 loctobre 1976), p. 223.

8. Voir, par exemple, sur l'affaire du Bill 63, J.C. Robert, op. cit., p. 257.

9. G. Marcotte, op. cit., p. 231. 
10. La composante amérindienne des origines de Marguerite renforce le mythe d'un peuple quê bécois uni en impliquant que ce peuple a su s'enrichir par l'intégration harmonieuse des Amérindiens. La mise en roman de cette intégration s'opère en partie à l'aide - le procédé est intéressant du point de vue du processus de l'écriture mythologique dans ce roman d'une ré-activation du mythe dix-huitiemiste du bon sauvage (l'Abénaki des pages 158-159). En outre, ce mythe de l'intégration harmonieuse a vraisemblablement pour fonction de légitimer le mythe-projet fondamental de l'édification d'un pays-nation québécois, en suggérant que cette édification ne nuira pas aux propriétaires premiers de l'espace québécois.

11. Cf. L'Hérault, op. cit., p. 92, et Gérard Tougas, Destin fittéraire du Québec, Montréał, Ėditions Québec/Amérique, 1982, p. 104.

12. P. L'Hérault, op. cit., p. 96.

13. Cf. Ibid., p. 104.

14. A en juger par lä bonne analyse de la substance idéologique des Grands Soleils qu'a donnée Ėlaine F. Nardocchio dans son article "Dimensions socio-politiques dans les Grands Soleils de Jacques Ferron" (Présence francophone, no 22 (printemps 1981), p. 131-140), Je SaintElias (1972) va plus loin dans le sens d'une idéologie dynamique et positive que ne le fait la pièce les Grands Soleils (1968), et traduit par là, nous semble-t-il, une évolution dans la pensée idéologico-politique de Jacques Ferron.

15. Anne Thérèse Reilly, "Présence du conte dans Cotnoir et le Saint-Elias de Jacques Ferron", mémoire de M.A., Vancouver, University of British Columbia, 1979, p. 28.

16. A. Renaud, op. cit., p. 44.

17. Cl. P. L'Hérault, op. cit., p. 243 sur les rapports entre fiction et réalité dans l'oeuvre ferronienne dans son ensemble.

18. Notre emploi du substantif "façonnage" ici repose sur deux des sens qu'en donne le Petit Robert (éd. 1977): "Action ou manière de façonner un ouvrage; son résultat", et aussi, "action de tirer des produits utiles des arbres abattus". 